\title{
Shuttling of (deoxy-) purine nucleotides between compartments of the diatom Phaeodactylum tricornutum
}

\author{
Lili Chu ${ }^{1}$, Ansgar Gruber ${ }^{1}$, Michelle Ast ${ }^{2}$, Stephan Schmitz-Esser ${ }^{3}$, Jacqueline Altensell ${ }^{2}$, Horst Ekkehard Neuhaus ${ }^{2}$, \\ Peter G. Kroth ${ }^{1}$ and Ilka Haferkamp ${ }^{2}$
}

${ }^{1}$ Pflanzliche Ökophysiologie, Fachbereich Biologie, Universität Konstanz, 78457 Konstanz, Germany; ${ }^{2}$ Pflanzenphysiologie, Technische Universität Kaiserslautern, 67653 Kaiserslautern,

Germany; ${ }^{3}$ Department of Animal Science, Iowa State University, Ames, IA 50011, USA

Key words: complex plastid, diatom, endoplasmic reticulum (ER), energy, nucleotide synthesis, nucleotide transport.

\section{Summary}

- Diatom plastids show several peculiarities when compared with primary plastids of higher plants or algae. They are surrounded by four membranes and depend on nucleotide uptake because, unlike in plants, nucleotide de novo synthesis exclusively occurs in the cytosol. Previous analyses suggest that two specifically adapted nucleotide transporters (NTTs) facilitate the required passage of nucleotides across the innermost plastid membrane. However, nucleotide transport across the additional plastid membranes remains to be clarified.

- Phylogenetic studies, transport assays with the recombinant protein as well as GFP-based targeting analyses allowed detailed characterization of a novel isoform (PtNTT5) of the six NTTs of Phaeodactylum tricornutum.

- PtNTT5 exhibits low amino acid similarities and is only distantly related to all previously characterized NTTs. However, in a heterologous expression system, it acts as a nucleotide antiporter and prefers various (deoxy-) purine nucleotides as substrates. Interestingly, PtNTT5 is probably located in the endoplasmic reticulum, which in diatoms also represents the outermost plastid membrane.

- PtNTT5, with its unusual transport properties, phylogeny and localization, can be taken as further evidence for the establishment of a sophisticated and specifically adapted nucleotide transport system in diatom plastids.

\section{Introduction}

All three main lineages of photosynthetic organisms, glauco phytes, rhodophytes, and chlorophytes (including land plants), according to the best supported hypothesis, gained their plastids in a process called primary endosymbiosis. During this primary endosymbiosis, a cyanobacterium was engulfed, retained and par tially degraded by a heterotrophic host (Keeling, 2013). Massive transfer of cyanobacterial genes to the host nucleus as well as metabolic connection and rearrangement of the consortium resulted in control and functional embedding of the symbiont and finally in the establishment of the photosynthetic organelle (Archibald, 2015). Interestingly, a considerable number of the diverse algal lineages arose from secondary endosymbioses. These processes occurred several times and included the fusion, and thus the genetic and metabolic connection, of two eukaryotes, a host cell and an alga with a primary plastid (Keeling, 2013; Archibald, 2015).

Diatoms are an important group of algae with secondary plas tids. They are of ecological relevance and of particular scientific interest because they are highly abundant in the oceans, and they are among the most common species of the phytoplankton and contribute immensely to carbon fixation (Falkowski \& Oliver,
2007; Armbrust, 2009). Genome analyses suggest that their rapid diversification, enormous success and predominance in the marine ecosystem might result from an extraordinary genetic composition/flexibility (Bowler et al., 2008; Armbrust, 2009; Archibald, 2015).

The complex plastids of diatoms are of red algal origin and possess four surrounding membranes of which the outermost is continuous with the rough endoplasmic reticulum (ER) (Kroth, 2002; Gould etal., 2008). Compared with plants, diatoms exhibit interesting metabolic peculiarities. For instance, nucleotide de novo synthesis is cytosolic in diatoms (Ast et al., 2009), whereas in higher plants, the complete pathway of purine and several steps of pyrimidine nucleotide synthesis take place within the plastid (Zrenner etal., 2006; Witz et al., 2012). Accordingly, in diatom plastids, nucleotides have to be trans ported across additional membranes and purine nucleotides even in an opposite direction (Ast et al., 2009) compared with higher plant plastids (Zrenner et al., 2006; Witz et al., 2012).

Previous studies indicate that at least two nucleotide trans porter (NTT) type carriers mediate nucleotide provision to the diatom plastids (Ast et al., 2009), whereas, in higher plant plas tids, transport of purine nucleotides and intermediates of pyrim idine nucleotide synthesis apparently involves different types of 
carriers (Leroch et al., 2005; Kirchberger et al., 2008; Witz et al., 2012).

Generally, NTT proteins are restricted to only a few organismic groups; they exist in certain obligate intracellular living bacteria and represent a hallmark of the orders Chlamydiales and Rickettsiales (Schmitz Esser etal., 2004). Chlamydiales and Rickettsiales are metabolically impaired (Moulder, 1991; Moran, 2002), lack several anabolic pathways, including de novo nucleotide synthesis, and exploit the nucleotide and energy pool of the host via interaction of functionally diverse NTT type proton symporters and exchangers (Krause et al., 1985; Tjaden et al., 1999; Haferkamp etal., 2004, 2006; Audia \& Winkler, 2006; Knab et al., 2011).

Among eukaryotes, NTTs have been detected in algae (with pri mary and secondary plastids) and higher plants (Möhlmann et al., 1998; Tjaden etal., 1998b; Linka et al., 2003; Tyra etal., 2007; Ast etal., 2009) and in protists of the phylum Microsporidia (Tsaousis et al., 2008; Heinz et al., 2014), whereas other protists, fungi and animals apparently lack such $n t t$ sequences in their genomes. NTTs of primary plastids were shown to operate exclu sively as ATP/ADP exchangers (Möhlmann et al., 1998; Tjaden et al., 1998b; Linka etal., 2003) in the inner plastid envelope. Analyses of mutant plants clearly demonstrated their role in energy provision to the stroma, particularly when photosynthesis is insuf ficient or missing (Tjaden et al., 1998a; Geigenberger et al., 2001; Reiser etal., 2004; Reinhold etal., 2007). Microsporidial NTTs reside in the plasma membrane or in the mitosome (a mitochon drial relict unable to produce energy in the form of ATP) and mediate ATP/ADP exchange or purine nucleotide transport (Tsaousis et al., 2008; Heinz et al., 2014).

Although, in recent years, the biochemical characteristics and physiological relevance of NTTs from several organisms have been revealed, our knowledge of NTTs from complex plastids is com paratively limited. This is because (1) metabolite transport into and/or out of complex plastids has not been investigated in detail, (2) diatoms possess an unusually high number of NTTs (six to eight isoforms) compared with other algae or higher plants (one to three isoforms), and finally (3) the two NTTs from diatoms char acterized to date (NTT1 and NTT2) exhibit functional properties previously not documented for any plastidial NTT and only rarely shown for NTTs from other organisms.

NTT1, and probably also NTT2, are located in the innermost envelope of the diatom plastid (Ast et al., 2009). The diatom NTT1 isoform acts as a proton symporter and accepts mono, $\mathrm{di}$, and triphosphorylated adenine nucleotides as substrates, whereas the NTT2 isoform transports various triphosphorylated nucleotides, including deoxy forms, in a counter exchange mode. Physiological interaction of NTT1 and NTT2 has been suggested to deliver all nucleotides for DNA and RNA synthesis to the stroma. Although NTT1 and NTT2 proteins represent impor tant components of the plastidial nucleotide uptake system in centric (Thalassiosirapseudonana) and pennate (Phaeodactylum tricornutum) diatoms, until now it has not been clarified how all required (deoxy) nucleotides pass through the remaining membranes of the complex plastid (Ast et al., 2009).

In this context, it is important to keep in mind that nucleotide transport can connect the sites of nucleotide synthesis and consumption, and/or play a key role in the adjustment of the sub cellular energy states. Just recently, plastids and mitochondria of diatoms were shown to be extraordinarily tightly connected by extensive energetic interaction (Bailleul etal., 2015). This metabolic communication relies on the transport of reducing power from plastids to mitochondria as well as on re shuttling of energy (from mitochondria to plastids), representing a main fac tor in the adaptation of cellular ATP/NADPH ratios for opti mized photosynthetic performance, carbon fixation and growth (Bailleul et al., 2015). Accordingly, the analysis of putative NTT proteins will help to deduce their possible role in nucleotide and/ or energy metabolism.

In this study, we focused on NTT isoform 5 from the diatom P. tricornutum (PtNTT5). PtNTT5 shows comparatively weak relationships to all previously characterized NTTs and here we report that it is also extraordinary in terms of its biochemical properties and subcellular localization.

\section{Materials and Methods}

\section{In silico analyses of NTT proteins}

Comparative amino acid sequence analysis of NTT proteins was performed using ClustalX (Thompson et al., 1997) and GenEDOC (Nicholas et al., 1997). Presence or absence of putative pre sequences, signal peptides, cleavage sites, chloroplast transit pep tides or transmembrane domains was investigated using the pro grams SignalP 3.0, ChloroP 1.1, TargetP 1.1, TMhmm (http:// www.cbs.dtu.dk/services/) (Emanuelsson etal., 2007) and ASAFIND (http://rocaplab.ocean.washington.edu/tools/asafind) (Gruber et al., 2015) following the methods described by Gruber \& Kroth (2014).

NTT amino acid sequences were aligned with MAFFT (Katoh \& Standley, 2013), and phylogenetic trees were reconstructed with Mega6 (Tamura et al., 2013) using the neighbor joining method and Poisson correction, the parsimony bootstrap method, and the maximum likelihood method (using the Jones-Taylor-Thornton (JTT) amino acid substitution model); all trees were calculated with $1000 \times$ bootstrapping. All positions containing gaps and missing data were eliminated. There were a total of 363 positions in the final data set.

\section{Cultivation of Phaeodacty/um tricornutum}

Phaeodactylum tricornutum Bohlin 1897, strain 646 (available at UTEX Culture Collection of Algae, University of Texas, Austin, TX, USA; https://utex.org) was cultivated in $\mathrm{f} / 2$ medium at a concentration of $50 \%$ sea water (16.6 g of sea salt; Tropic Marin Dr. Biener, GmbH, Wartenberg, Germany) in 11 of dis tilled water, adjusted to $\mathrm{pH}$ 7.0. Supplements were added as described in Kroth (2007). For agar plate cultivation, corre sponding solid media were complemented with $1.2 \%$ Bacto Agar (Becton, Dickinson \& Co., Le Pont de Claix, France). Cells were grown under continuous illumination at $35 \mu \mathrm{mol}$ photons $\mathrm{m}^{-2} \mathrm{~s}^{-1}$ or with a day-night cycle $(16 \mathrm{~h}: 8 \mathrm{~h}$, light : dark; for protein preparation and western blotting). 


\section{Preparation of cDNA and generation of expression constructs}

RNA isolation was performed with the RNeasy plant mini kit (Qiagen, Hilden, Germany) and poly $\left(\mathrm{A}^{+}\right) \mathrm{RNA}$ was enriched from this preparation with the Oligotex kit (Qiagen). Production of cDNA from mRNA templates was conducted with the reverse transcriptase SuperscriptII (Invitrogen, Carlsbad, CA, USA) according to the manufacturer's instructions. The coding sequence of PANTT5 was amplified via PCR and oligonucleotides (MWG, Ebersberg, Germany or Sigma Aldrich, Munich, Germany) that allow compatible insertion into the expression vectors. The ampli fication products were restricted with specific endonucleases, puri fied from the agarose gel with the NucleoSpin Extract II Kit (Macherey \& Nagel, Düren, Germany).

For generation of the Escherichia coli expression construct, the coding sequence of full length PtNTT5 was inserted via the restriction sites $N d e I$ and $X h o I$ in frame with the histidine tag into the Isopropyl $\beta$ D thiogalactoside inducible expression vec tor pET16b (Novagen, Heidelberg, Germany).

For the localization studies, the start codon of the eGFP gene of the pPha T1 GFP plasmid (Gruber et al., 2007) (a modified version of the shuttle vector pPha T1; GenBank AF219942; Zaslavskaia et al., 2000) was replaced by the codon GGA (coding for glycine) via site directed mutagenesis to inactivate the poten tial translation start. The PtNTT5 amplicon was ligated into the Stul site of this modified pPha T1 GFP plasmid. Truncated ver sions of PANTT5 were produced via PCR or deletion PCR with specific oligonucleotides (Supporting Information Table S1) and using the vector containing full length PtNTT5 with either N or $\mathrm{C}$ terminal GFP fusion as a template.

Amplification products were purified and either inserted into the pPha T1 shuttle vectors or re ligated (deletion PCR prod ucts). Correctness of the respective constructs was verified by restriction analyses (Fermentas, St Leon Rot, Germany) and sequencing (SEQ IT, Kaiserslautern, Germany or GATC Biotech AG, Konstanz, Germany).

\section{Heterologous expression in Escherichia coli and import measurements}

The E. coli strain Rosetta 2(DE3)pLysS (Novagen) was used for heterologous synthesis of PtNTT5. To analyze transport proper ties of the recombinant PtNTT5, either induced or noninduced E. coli cells harboring the expression plasmid pET16b PtNTT5 were incubated in phosphate buffer $\left(\mathrm{KP}_{\mathrm{i}}\right)$ supplemented with the respective $\left[\alpha^{32} \mathrm{P}\right]$ labeled substrates. Import was conducted at $30^{\circ} \mathrm{C}$ and terminated by removal of external substrate using vac uum filtration and washing. Radioactivity was quantified by scin tillation counting (Tricarb 2500; Canberra Packard, Heidelberg, Germany).

\section{Nuclear transformation and microscopy}

Nuclear transformation of P. tricornutum was performed as described previously (Kroth, 2007). Cellular localization of GFP fusion proteins was analyzed with confocal laser scanning micro scope LSM 780 or LSM 510 META (Carl Zeiss, Oberkochen, Germany) using a Plan Apochromat $63 \times 1.4$ oil immersion Nomarski differential interference contrast (DIC) objective (Carl Zeiss) or an epifluorescence microscope Olympus BX51 (Olym pus Europe, Hamburg, Germany) equipped with a Zeiss AxioCam MRm digital camera (Carl Zeiss) and an Olympus PLN $\times 40$ objective (Olympus). Nucleic acids were stained with the dye Hoechst 33342 (Calbiochem ${ }^{\circledR}$; Behring Diagnostics, La Jolla, CA, USA) to indicate the location of the nucleus, while MitoTracker ${ }^{\circledR}$ Orange CM H $\mathrm{H}_{2}$ TMRos (Molecular Probes, Inc., Eugene, OR, USA) showed the location of the mitochondria. Image processing was conducted using ZEN LITE and AxIOVISION REL. 4.7 (Carl Zeiss).

\section{Western blot analyses and SDS-PAGE}

Cells were harvested during the exponential growth phase by cen trifugation $\left(3000 \mathrm{~g}\right.$ for $10 \mathrm{~min}$ at $4{ }^{\circ} \mathrm{C}$ ) and the pellet was resus pended in $1 \mathrm{ml}$ of lysis buffer containing protease inhibitor 'complete EDTA free' (Roche, Mannheim, Germany), $50 \mathrm{mM}$ Tris $\mathrm{HCl}, \mathrm{pH} 8,1 \mathrm{mM}$ EDTA, $8 \mathrm{M}$ urea and 1\% SDS. A mix ture of glass beads $(0.1-1 \mathrm{~mm}$ diameter) was added and cells were homogenized in Fast Prep FP 120 Bio 101 Savant (Qbio gene Inc., Carlsbad, CA, USA) at maximum speed six times for $20 \mathrm{~s}$ each, being kept on ice for $1 \mathrm{~min}$ between each homogeniza tion. Samples were again centrifuged to remove nondisrupted cells. The total protein concentration in the supernatant was determined using the $660 \mathrm{~nm}$ Pierce Protein Assay (Thermo Sci entific, Schwerte, Germany). Five micrograms of this protein extract was analyzed in a discontinuous, denaturing system on $10 \%$ or $12 \%$ separating polyacrylamide gels (Laemmli, 1970) and further transferred onto a nitrocellulose membrane (What man Protran BA 79; Whatman Inc., Maidstone, UK) elec trophoretically using a Trans Blot Turbo (Bio Rad, Hercules, CA, USA). The Page Ruler Prestained Protein Ladder (Thermo Scientific) was used as a marker.

Immunodetection was performed using an anti GFP antibody (Invitrogen, Molecular Probes, Eugene, OR, USA) combined with anti Rabbit IgG peroxidase antibody (Sigma Aldrich). The western blots were treated with $\operatorname{Roti}^{(}{ }^{\circledR}$ Block, Roti ${ }^{\circledR}$ Blue and Roti ${ }^{\circledR}$ Lumin plus, as described in the manuals (Carl Roth GmbH \& Co. KG, Karlsruhe, Germany). Chemoluminescence was documented with Amersham Hyperfilm ECL (GE Health care, Little Chalfont, UK) and Konica SRX 201 (Konica Minolta, Chiyoda, Japan).

\section{Results}

\section{The evolutionary origin of PtNTT5}

To elucidate the possible evolutionary origin of diatom NTTs, we performed a phylogenetic analysis with published and bio chemically characterized NTTs from various organisms as well as NTT isoforms from Thalassiosira pseudonana (TpNTT1 8) and P. tricornutum (PtNTT1 6). 
The overall topology of the obtained phylogenetic tree (Fig. 1) is in agreement with previous studies performed with larger data sets including also various NTTs of unknown function (Tyra et al., 2007; Tsaousis etal., 2008; Ast etal., 2009; Heinz et al., 2014).

Microsporidial isoforms generally constitute a separate branch among the NTTs (Fig. 1; Tsaousis etal., 2008; Heinz et al., 2014). The isoforms of Trachipleistophora hominis and Encephalitozoon cuniculi form two distinct clusters. This is in line with recent analyses suggesting that the different NTT isoforms of contemporary microsporidians arose from repeated, lineage specific gene duplications whereas the ancestor of microsporidians probably possessed only a single NTT gene (Heinz et al., 2014).

It is widely accepted that early primary photosynthetic eukaryotes probably acquired the nucleotide transporter gene from a donor closely related to extant chlamydiae (Greub \& Raoult, 2003; Schmitz Esser etal., 2004; Tyra et al, 2007), and also in our analysis a monophyletic grouping of plastidial and chlamydial ATP/ADP transporters (bacterial NTT1 isoforms) was evident (Fig. 1). Moreover, plastidial NTTs from higher plants, red algae and diatom NTT1 isoforms form a mono phyletic cluster and their close relationship as sister groups sug gests that diatom NTT1 proteins originated from the red algal

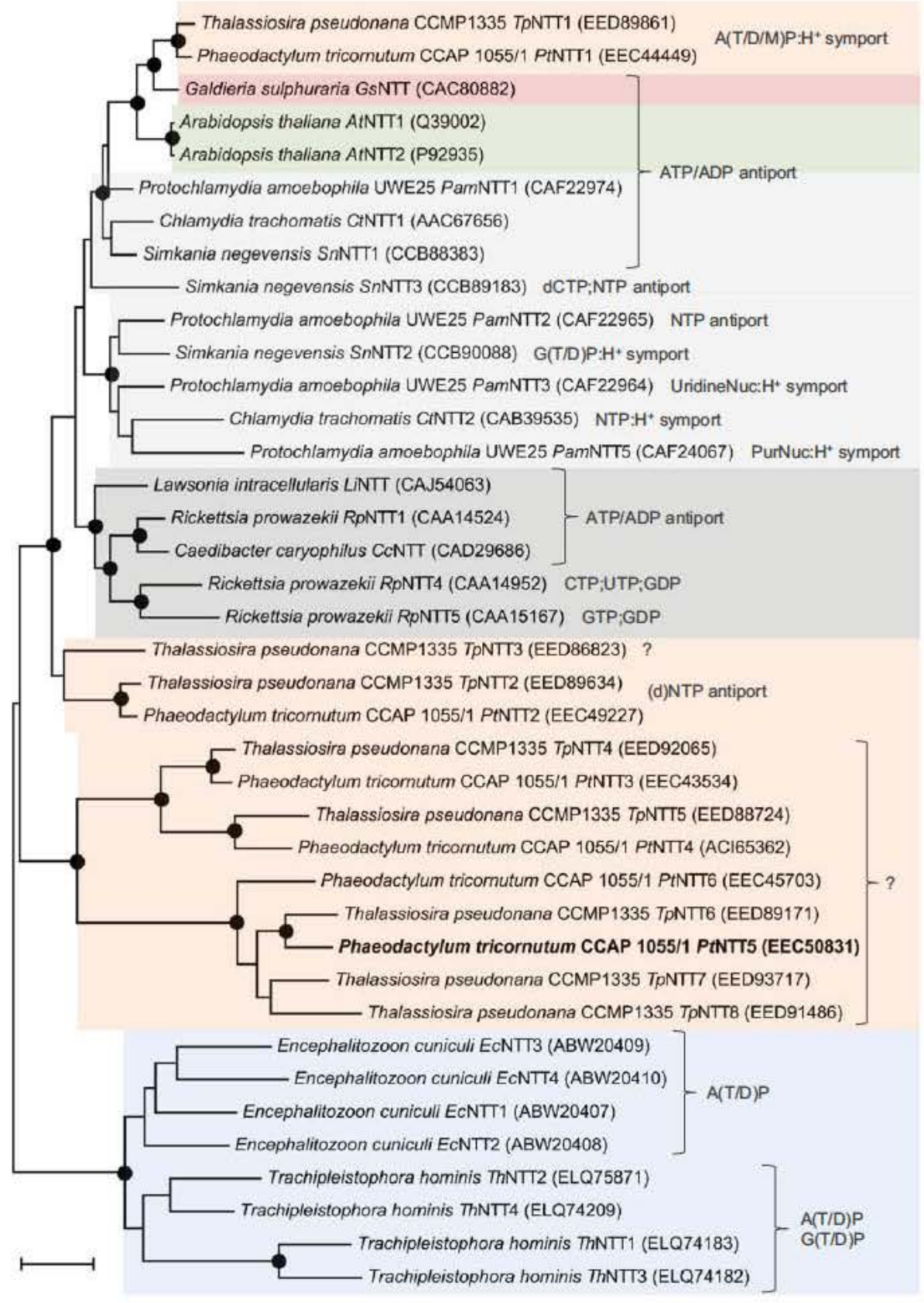

Fig. 1 Phylogenetic relationships of nucleotide transporters (NTTs) from various organisms. An amino acid based phylogenetic tree calculated with MEGA6 using the maximum likelihood algorithm with the Jones-Taylor-Thornton (JTT) model is shown. Black dots indicate nodes supported by maximum likelihood, maximum parsimony, and neighbor joining bootstrap values $(1000 \times$ resampling $)>90 \%$. GenBank accession numbers are given in brackets. The bar represents $20 \%$ estimated evolutionary distance. Organismic groups are marked by shading: diatoms, orange; red alga, red; higher plants, green; Chlamydiales, Rickettsiales and Lawsonia intracellularis, grayscales; microsporidia, blue. Basic transport features were taken from Schmitz Esser et al. (2004), Audia \& Winkler (2006), Schmitz Esser etal. (2008), Ast etal. (2009), Knab et al. (2011), Heinz et al. (2014) and literature therein. 
progenitor engulfed during secondary endosymbiosis (Fig. 1; Ast et al., 2009).

Diatom NTT2 proteins and TpNTT3 form a deep branch together with functionally diverse NTTs from rickettsiae (Ast et al., 2009) or even constitute a separate group among various bacterial and plastidial NTTs (Fig. 1). The remaining diatom NTT isoforms also form a distinct subgroup of NTTs with two well supported subclusters (Fig. 1). One subcluster is formed by $P t$ NTT3, PtNTT4 and the respective homologs from T. pseudonana, TpNTT4 and TpNTT5. PtNTT5 and its possible homolog TpNTT6 constitute the second subcluster together with PtNTT6, TpNTT7 and TpNTT8. Therefore, these diatom NTTs seem to be only weakly related to other bacterial and plas tidial NTTs and only the cluster formed by the microsporidial NTTs exhibits a higher phylogenetic distance.

The branching pattern indicates that the diatom NTT1 iso form originated from endosymbiotic gene transfer, whereas the ancestor of the remaining diatom NTTs cannot be resolved. It is possible that those proteins arose from the red algal derived NTT1 isoform via several gene duplication events. If this is the case, the origin of these isoforms is apparently masked by consid erable alterations in the amino acid sequences, maybe as a conse quence of functional constraints. Alternatively, the remaining diatom NTTs may have resulted from horizontal gene transfer from bacterial (or maybe also microsporidial) donors, and subse quent gene duplications led to the establishment of the respective subgroups of NTTs in diatoms. Their distant relationship to the plant NTTs, including the diatom NTT1 isoforms, and the fact that horizontal gene transfer is an important and common phenomenon in diatom evolution, might be taken to support the second scenario (Bowler et al., 2008).

In fact, the nuclear genome of diatoms appears to be a patch work and comprises a comparatively high number of genes from different bacterial sources (Bowler et al., 2008). The higher GC content of PtNTT5 and PtNTT6 (Table S2), but most impor tantly the different numbers of introns (Fig. S1) suggest that the remaining NTT isoforms (with no or, in a few cases, one or two introns) do not represent modified duplications of NTT1 (with three introns), but instead were gained by at least one or by several independent horizontal gene transfers. The intron number of putative homologs also differs between the two diatom species and suggests a slightly higher tendency for intron gain in $T$. pseudonana. This is in line with comparative genome analyses documenting a higher number of introns in $T$. pseudonana com pared with $P$. tricornutum (Bowler et al., 2008). The diatom iso forms PtNTT3 6 and TpNTT4 8 are phylogenetically clearly separated from the previously characterized NTTs. This is why we were interested in a detailed analysis of PtNTT5 as a first rep resentative member of this particular group of diatom NTTs.

\section{Peculiarities in the amino acid sequence of PtNTT5}

From our sequence alignment of PtNTT5 and representative, biochemically characterized NTTs from diverse organisms, it became evident that the amino acid sequences of NTTs from plants and algae possess an $\mathrm{N}$ terminal extension compared with the remaining NTTs (Fig. S2). These extensions generally con tain information supporting plastid targeting. In diatoms, nuclear encoded proteins with a stromal destination exhibit a characteristic bipartite arrangement of the $\mathrm{N}$ terminal extension (pre sequence) starting with a signal peptide followed by a transit peptide. Moreover, a conserved motif, the so called 'ASAFAP' motif, mostly flanks/overlaps with the predicted signal peptide cleavage site (Kilian \& Kroth, 2005; Gruber et al., 2007). NTT1 and NTT2 from diatoms do show such a typically structured N terminal targeting sequence and NTT1 isoforms even contain an authentic 'ASAFAP' motif at the signal peptide (Fig. S2; Ast et al., 2009).

PtNTT5 lacks a comparable $\mathrm{N}$ terminal extension and in this context rather resembles microsporidial NTTs which are mainly located in the plasma membrane. In silico analyses also failed to detect a possible $\mathrm{N}$ terminal signal peptide with a corresponding peptide cleavage site (Ast et al., 2009). Moreover, prediction of the subcellular localization of PtNTT5 was unclear (Ast et al., 2009). However, the absence of characteristic plastidial targeting motifs (Fig. S2; Ast et al., 2009) implies that PtNTT5 resides in a different compartment/membrane from isoforms 1 and 2 . A detailed inspection of the PtNTT5 sequence revealed a compara tively high accumulation of positively charged amino acid residues at its far $\mathrm{C}$ terminus; however, so far it is unclear whether this finding is of any relevance for correct protein target ing or membrane insertion.

PtNTT5 generally exhibits only moderate amino acid sequence similarities (24-31\%) to previously described NTTs, including diatom NTT1 and NTT2 isoforms (Table S3). Several regions that are conserved in plant and bacterial NTTs show higher variations in PtNTT5 as well as in microsporidial NTTs (Fig. S2). Moreover, not all predicted transmembrane domains of PtNTT5 did match with the hydrophobic regions of represen tative characterized NTTs. Based on sequence similarities, it is hard to judge whether PtNTT5 is involved in plastidial nucleotide transport or whether it even fulfills a different physio logical function.

\section{PtNTT5 accepts various purine nucleotides as substrates}

We determined the functionality and biochemical properties of PtNTT5 using transport measurements with radioactively labeled substrates and $E$. coli cells expressing the recombinant carrier. Ini tial studies with $\left[\alpha^{32} \mathrm{P}\right]$ ATP and $\left[\alpha^{32} \mathrm{P}\right]$ ADP revealed that PtNTT5 in fact is able to transport nucleotides (Fig. 2). ATP and ADP were efficiently imported into E. coli via PtNTT5, whereas noninduced cells (control) showed no or only marginal accumu lation of radioactively labeled nucleotides.

To determine the general substrate specificity of PtNTT5, we conducted competition experiments with different nonlabeled nucleotides or nucleotide derivatives. In addition to nonlabeled ATP and ADP, dATP and AMP, as well as the corresponding guanidine nucleotides, markedly reduced $\left[\alpha^{32} \mathrm{P}\right]$ ATP uptake (Table 1). Among the tested purine nucleotides, GDP addition resulted in the lowest decrease of $\left[\alpha^{32} \mathrm{P}\right]$ ATP uptake, and the corresponding reduction was similar to that caused by TTP, but 
(a)

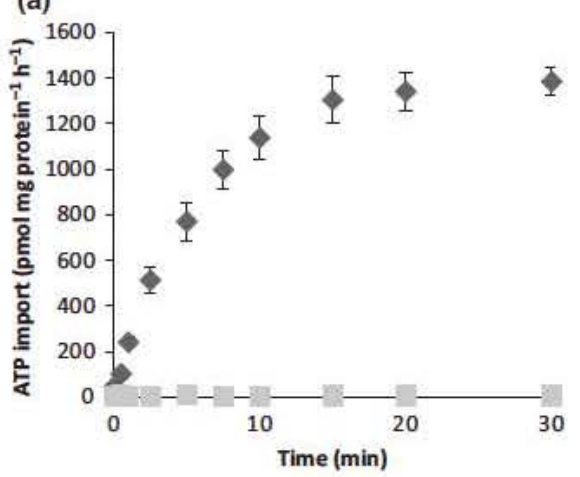

(b)

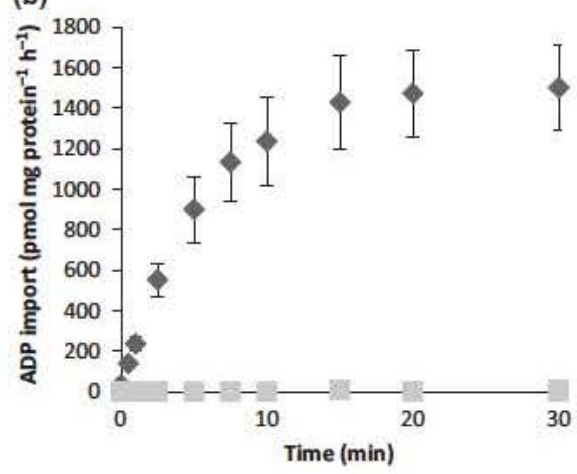

Fig. 2 Time dependent uptake of ATP and $A D P$ via nucleotide transporter isoform 5 from Phaeodactylum tricornutum (PtNTT5). Transport of $\left[\alpha^{32} \mathrm{P}\right]$ ATP $(\mathrm{a})$ and $\left[\alpha^{32} \mathrm{P}\right]$ ADP (b) into Isopropyl $\beta$ D thiogalactoside induced Escherichia coli cells synthesizing PtNTT5 (black symbols) in comparison to nucleotide uptake into corresponding noninduced $E$. coli cells (gray symbols) is shown. Data are the mean of at least three independent experiments, $\pm \mathrm{SE}$.
Table 1 Effects of various nucleotides and nucleotide derivates on $\left[\alpha^{32} \mathrm{P}\right]$ ATP import via nucleotide transporter 5 from Phaeodactylum tricornutum (PtNTT5)

\begin{tabular}{llllcr}
\hline Effector & Import $(\%)$ & SE $(\%)$ & Effector & Import $(\%)$ & SE (\%) \\
\hline$(-)$ & 100 & 0 & CDP & 71.5 & 2.9 \\
ATP & 26.0 & 1.0 & TMP & 99.7 & 4.5 \\
ADP & 28.3 & 2.7 & dATP & 33.9 & 2.2 \\
AMP & 21.7 & 1.7 & dGTP & 30.1 & 1.8 \\
GTP & 43.0 & 1.5 & dCTP & 70.0 & 1.8 \\
GDP & 53.6 & 1.8 & TTP & 50.5 & 6.2 \\
GMP & 37.5 & 2.9 & CAMP & 104.5 & 5.3 \\
ITP & 67.1 & 3.2 & CGMP & 91.2 & 4.2 \\
IDP & 81.9 & 3.4 & NAD & 88.2 & 5.5 \\
IMP & 82.5 & 5.0 & PAP & 93.6 & 4.3 \\
UTP & 62.8 & 2.3 & PAPS & 97.8 & 11.5 \\
UDP & 81.6 & 3.4 & NADP & 95.2 & 6.2 \\
UMP & 96.7 & 3.4 & COA & 89.3 & 4.7 \\
CTP & 78.0 & 3.6 & FAD & 88.5 & 3.0 \\
\hline
\end{tabular}

Uptake of $\left[\alpha^{32} \mathrm{P}\right]$ ATP by recombinant PtNTT5 at a substrate concentration of $50 \mu \mathrm{M}$ was set to $100 \%$ (control). Corresponding transport in the pres ence of nonlabeled effectors ( 10 fold excess) was calculated according to the control. Import was stopped after $3 \mathrm{~min}$. Nucleotide uptake rates are net values (minus control: ATP uptake into noninduced Escherichia coli cells) and the mean of three independent experiments. Bold type is used to highlight significantly reduced import rates ( $<50 \%$ residual activity).

higher than that produced by UTP addition. To demonstrate that the given molecules do represent substrates competing with ATP for import, but are not inhibitors of the transport protein, we performed uptake studies with the corresponding radiolabeled isotopes. All tested nucleotides were imported and most of them exhibited rates comparable to the transport rates of ATP and ADP; however, GMP caused slightly lower, whereas deoxy purine nudeotides caused higher transport rates (Fig. S3). There fore, a broad set of purine nucleotides, including dATP and dGTP, were identified as substrates of PANTT5.

To examine the biochemical properties of PtNTT5 in more detail, we determined the substrate affinities (Michaelis constant, $\left.K_{\mathrm{M}}\right)$ as well as the corresponding maximal transport velocities $\left(V_{\max }\right)$. For this purpose, transport measurements were con ducted in the presence of rising concentrations of the respective labeled nucleotides (Table 2). PtNTT5 exhibited the highest affinities for adenine nucleotides (c. 120-150 $\mu \mathrm{M})$ followed by
dATP and dGTP (c. $210 \mu \mathrm{M})$, whereas guanidine nucleotides were generally imported with lower affinities (c. 310-350 $\mu \mathrm{M}$ ). GDP and ATP were imported with the lowest maximal velocity (c. 30 and $37 \mathrm{nmol} \mathrm{mg}^{-1}$ protein $\mathrm{h}^{-1}$, respectively) compared with the remaining (deoxy) nucleotides (c. 60-84 $\mathrm{nmol} \mathrm{m}{ }^{-1}$ protein $\mathrm{h}^{-1}$ ). These results demonstrate that not only tri, di, and monophosphorylated adenine and guanidine nucleotides, but also dATP and dGTP represent relevant sub strates of $P t$ NTT5.

\section{PtNTT5 is an antiporter}

To reveal the NTT function in the cellular context, it is impor tant to determine its mode of transport. In principle, NTT type proton symporters enable net gain of nucleotides, whereas NTT type antiporters either allow energy uptake by the exchange of ATP and ADP or the adaptation of the composition of the respective nucleotide pools by exchanging internal with external (deoxy) nucleotides.

To analyze whether recombinant $P t$ NTT5 is driven by the proton gradient across the $E$. coli membrane, we investigated the influence of the protonophore $\mathrm{m}$ chlorophenylhydrazone (CCCP) on ATP import (Fig. 3a). Interestingly, nudeotide import was not inhibited, but rather slightly stimulated by mod erate CCCP concentrations (at 50-100 $\mu \mathrm{M} \mathrm{CCCP:} \mathrm{transport}$ rate $>100 \%$ ), while rather high concentrations $(>250 \mu \mathrm{M}$ CCCP) were required to cause a visible reduction of transport compared with unaffected transport (set to $100 \%$ ). Even in the presence of $750 \mu \mathrm{M}$ CCCP, PANTT5 showed quite high trans port activity of $68 \%$ (inhibition by $32 \%$ ). Proton driven NTTs are generally known to be highly inhibited (by $65-80 \%$ ) already at moderate CCCP concentrations (10-100 $\mu \mathrm{M}$ CCCP: trans port rates 20-35\%) (Haferkamp etal., 2006; Ast et al., 2009; Knab etal., 2011), and thus we can condude that PtNTT5 does not act as a proton symporter.

In a next step, we investigated the capacity of nonlabeled import substrates to induce export of radioactively labeled nucleotides (previously loaded into $E$. coli) in a chase experiment. Escherichia coli expressing PtNTT5 was incubated in $50 \mu \mathrm{M}$ $\left[\alpha^{32} \mathrm{P}\right]$ ATP and the radioactivity in the cells was quantified over time. After $5 \mathrm{~min}$ of import, significant amounts of $\left[\alpha^{32} \mathrm{P}\right]$ ATP accumulated in the cells and possible efflux was started by 
Table 2 Determination of apparent Michaelis constant $\left(K_{M}\right)$ and maximal velocity $\left(V_{\max }\right)$ values of purine nucleotide transport mediated by nucleotide transporter 5 PtNTT5 from Phaeodactylum tricornutum (PtNTT)

\begin{tabular}{|c|c|c|c|c|c|}
\hline \multirow[b]{2}{*}{ Substrate } & \multicolumn{2}{|c|}{ Adenine nucleotides } & \multirow[b]{2}{*}{ Substrate } & \multicolumn{2}{|c|}{ Guanidine nucleotides } \\
\hline & $K_{M}$ & $V_{\max }$ & & $K_{M}$ & $V_{\max }$ \\
\hline ATP & $120.7(13.4)$ & $36.8(3.7)$ & GTP & $311.5(19.6)$ & $73.6(9.6)$ \\
\hline ADP & $137.0(1.8)$ & $59.9(4.8)$ & GDP & $304.0(23.1)$ & $29.2(2.4)$ \\
\hline AMP & $151.0(8.9)$ & $71.9(8.3)$ & GMP & $348.0(31.0)$ & $83.8(9.1)$ \\
\hline dATP & $207.2(17.6)$ & $84.1(6.4)$ & dGTP & $211.3(26.7)$ & $82.1(6.3)$ \\
\hline
\end{tabular}

Nucleotide uptake in the presence of rising substrate concentrations $(51000 \mu \mathrm{M})$ was allowed for time spans in the linear phase of the corresponding transport at $50 \mu \mathrm{M}$. Apparent $K_{\mathrm{M}}$ values are given in $\mu \mathrm{M} ; V_{\max }$ values are given in $\mathrm{nmol} \mathrm{mg}{ }^{-1}$ protein $\mathrm{h}^{-1}$. Data are the mean of at least three independent experiments. Corresponding standard errors are given in brackets.
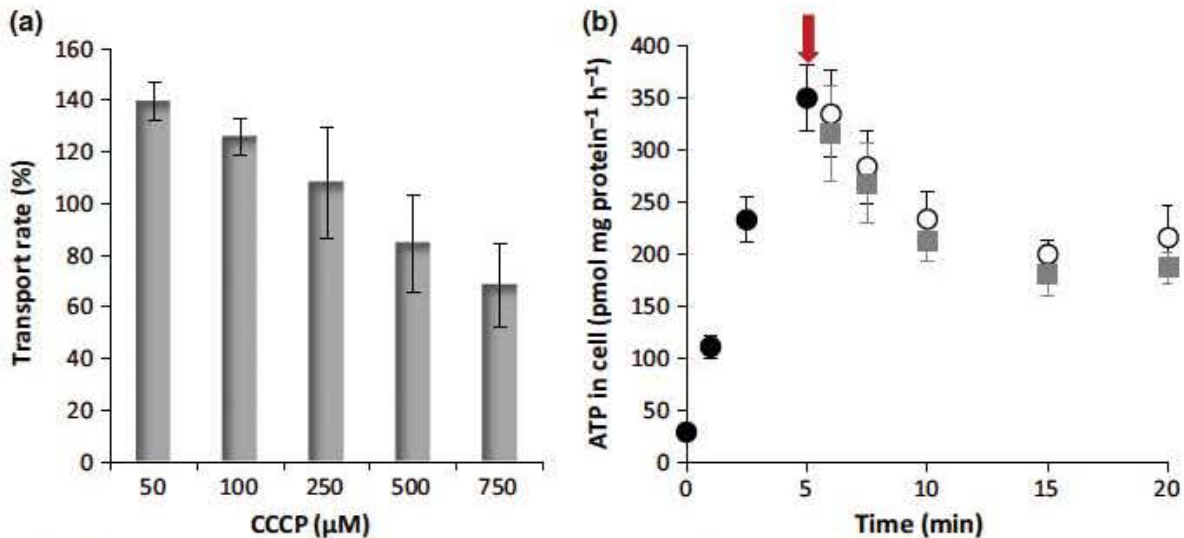

Fig. 3 Analysis of the transport mode of nucleotide transporter 5 (NTT5) from Phaeodactylum tricornutum. (a) Influence of membrane potential depletion by protonophore addition on the transport activity of PtNTT5. Unaffected net [ $\left.\alpha^{32} \mathrm{P}\right]$ ATP import (import into induced cells minus rates of noninduced cells) was set to $100 \%$. [ $\alpha^{32} \mathrm{P}$ ] ATP import in the presence of rising $\mathrm{m}$ chlorophenylhydrazone (CCCP) concentrations was calculated as a percentage of the unaffected transport. (b) Investigation of a possible counter exchange transport mode. Time dependent accumulation of $50 \mu \mathrm{M}$ ATP in Escherichia coli cells expressing PENTT5 is shown (black circles). After 5 min of ATP import, possible efflux of internal label was induced by the addition of a 20 fold excess of nonlabeled substrates (red arrow). Efflux induction was tested with GTP (white circles) and dGTP (gray squares) as representative counter exchange substrates. Data are the mean of at least three independent experiments, $\pm \mathrm{SE}$.

addition of nonlabeled GTP or dGTP. Internal radioactivity subsequently markedly decreased, indicating that uptake of non labeled nucleotides is accompanied by the export of interior label. This observation indicates that PtNTT5 operates in an antiport mode (Fig. 3b).

\section{GFP fusions suggest targeting of PtNTT5 to the ER mem- brane}

The amino acid sequence of PtNTT5 lacks the $\mathrm{N}$ terminal signal peptide typical of proteins with plastidial destination (stroma/ innermost membrane) and also in silico analyses failed to clearly predict a specific subcellular targeting. We therefore expressed different GFP fusion proteins of PtNTT5 in P. tricornutum to deduce the possible in vivo localization of the transporter. We fused GFP to either the $\mathrm{N}$ or the $\mathrm{C}$ terminus of PANT5. Fluo rescence of the $\mathrm{N}$ terminal GFP fusion labeled a network like structure distributed throughout the cell, surrounding the plastid and the nucleus (Fig. 4a,b). This fluorescence pattern is very char acteristic for proteins located in the continuous ER/chloroplast ER (cER) (Apt et al., 2002). As a reference protein for the ER, we re investigated a P. tricornutum cell line (generated by Apt et al.,
2002), which expresses GFP fused to the pre sequence of the ER luminal binding protein precursor (BIP) (Fig. S4). By staining intracellular DNA with the dye Hoechst 33342, we confirmed that the GFP signal (of the ER) surrounded the nucleus (Fig. 4a), as expected because of the continuity of the ER, nuclear envelope and cER lumen.

The C terminal GFP fusion protein did not label a cellular network, but instead accumulated in rather diffuse patches close to the plastid/nucleus (Fig. 4c). This pattern differed from that of ER targeted proteins (Fig. S4) and did not totally cover or sur round the chlorophyll autofluorescence area of the plastid. More over, it did not overlap with the MitoTracker signal, which argues against a potential mitochondrial localization.

To investigate the subcellular localization of PtNTT5 in inde pendent systems, we transiently expressed PtNTT5 as C terminal and $\mathrm{N}$ terminal GFP fusions (Grefen et al., 2010) in Nicotiana benthamiana and in Arabidopsis thaliana. Also in higher plant cells, PtNTT5 with N terminal GFP was targeted to a network like structure (Fig. S5) and the GFP signal overlapped with that of the co expressed ER marker protein (ER rk; Nelson et al., 2007). By contrast, PANTT5 with $C$ terminal GFP showed quite heterogeneous and ambiguous fluorescence patterns in both 
(a)

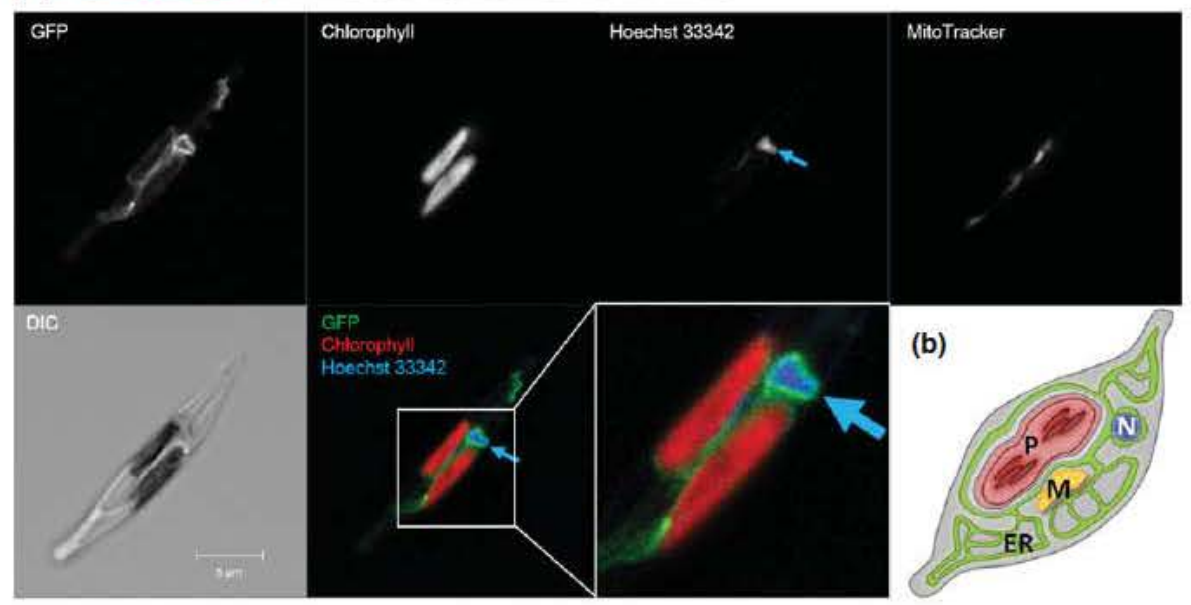

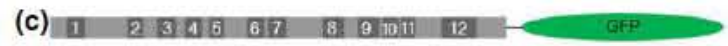

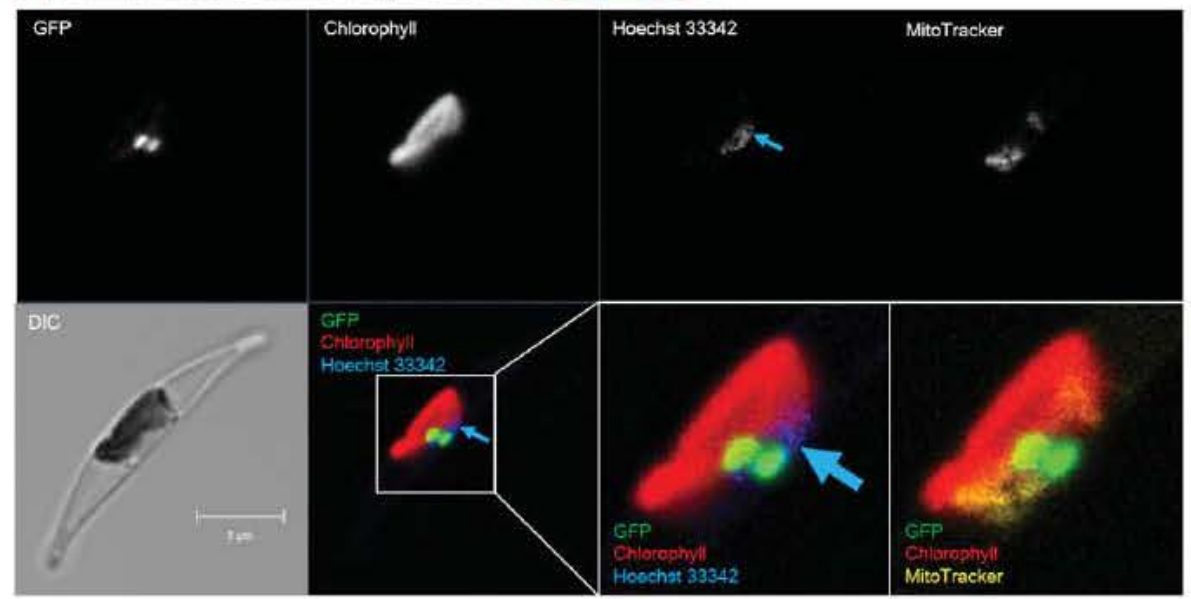

Fig. 4 Cellular localization of nucleotide transporter 5 (NTT5) in Phaeodactylum tricornutum cells. (a) GFP fused $\mathrm{N}$ terminally to PtNTT5. (b) Schematic illustration of a $P$. tricornutum cell depicting the stained organelles. (c) GFP fused C terminally to PtNTT5. GFP fluorescence, green; autofluorescence of the chlorophyll, red; Hoechst 33342 fluorescence, blue; MitoTracker fluorescence, yellow; Nomarski differential interference contrast (DIC), grayscale. The position of the Hoechst stain is marked with an arrow. Bars, $5 \mu \mathrm{m} . \mathrm{N}$, nucleus; $M$, mitochondrion; $E R$, endoplasmic reticulum; $P$, plastid. tobacco and $A$. thaliana (Fig. S6). Diffuse GFP signals and punc tuated structures appeared in the cytosol and at the nucleus. The fluorescence also surrounded the plastids, but in quite an irregu lar manner with alternating regions of higher and lower intensity. In contrast to PtNTT5 with N terminal GFP, which labeled the entire ER network, the apparent ER localization of the $C$ terminal GFP fusion protein was mainly restricted to the nuclear region and was not as constant and precise as observed with the $\mathrm{N}$ terminal GFP construct.

The finding that $\mathrm{N}$ and $\mathrm{C}$ terminal GFP constructs caused different fluorescence patterns led us to the assumption that the position of the marker protein affects subcellular targeting or membrane insertion. In order to identify possible targeting domains and to determine whether the $\mathrm{C}$ or the $\mathrm{N}$ terminal GFP fusion reflects the localization of the native transporter, we performed targeting experiments with truncated versions of PtNTT5 carrying GFP either at the $\mathrm{C}$ terminus or at the $\mathrm{N}$ terminus (Figs S7-S11).

Microscopy analyses of the respective transformed cell lines revealed that almost all fusion proteins caused GFP signals. Expression of selected GFP fusions was additionally monitored by western blotting and immuno staining using an GFP anti body. This method not only verified the successful expression of the various fusion proteins (including one construct that did not cause visible GFP fluorescence) but also documented their differ ent molecular weights (Fig. S12).

The fluorescence pattern resulting from the truncated versions of PtNTT5 with C terminal GFP resembled that of the corre sponding full length protein, no matter which part of the protein was deleted (Figs S8, S9). Therefore, no specific region(s) in the sequence of PtNTT5 could be detected that may have caused the accumulation of GFP in proximity to the plastid, suggesting that this localization might be attributable to the $\mathrm{C}$ terminal position of GFP. By contrast, $\mathrm{C}$ terminally truncated PATT5 proteins with $\mathrm{N}$ terminal GFP caused different labeling patterns. Accord ingly, the subcellular targeting of these fusion proteins depends on the length of the amino acid sequence of PtNTT5. The GFP signal caused by the shorter versions of PtNTT5 (up to 361 amino acids in length) appeared somewhere in the cytosol, whereas longer versions (at least 390 amino acids in length) were targeted to the (chloroplast) ER (Figs S10, S11). Thus, the longer the fragment, the more the labeling pattern resembled that of the corresponding full length protein.

These results suggest that adding GFP to the $C$ terminus of PtNTT5 may prevent targeting to the ER by masking an as yet unknown motif or a domain required for (chloroplast) ER 
targeting. Furthermore, both a (nearly) full length protein sequence and the unblocked $\mathrm{C}$ terminal part of PANTT5 may be essential for correct targeting. In this context, it is important to mention that potential targeting information within the protein sequence (particularly the remarkable accumulation of positive charges) at the far $\mathrm{C}$ terminus could not be clearly associated with ER targeting, because the correspondingly truncated PtNTT5 versions with $\mathrm{N}$ terminal GFP were also detectable in the ER.

\section{Discussion}

Diatoms possess the highest number of putative NTTs (up to eight) among all known organisms (Ast etal., 2009) which immediately raises questions regarding the phylogeny and physi ological benefits of the different isoforms.

In phylogenetic studies, diatom NTT1 isoforms have been found to be most closely related to ATP/ADP transporters from red algae (Fig. 1; Ast et al., 2009). Therefore, NTT1 was probably introduced into the diatom genome via gene transfer from the red algal endosymbiont. The remaining diatom NTTs form separate clusters, more distantly related to the plastidial ATP/ADP transporters from plants and algae (Fig. 1). Although the direct ancestor(s) of these NTTs cannot be iden tified, their low number of introns and the high GC content of at least PtNTT5 and PtNTT6 argue against a possible origin from the red algal derived NTT1 isoform by gene duplication and subsequent modification (Table S2; Fig. S1). The origin of almost all diatom NTTs (apart from NTT1 iso forms) by horizontal gene transfer from bacterial sources thus appears more likely. The branching pattern might indicate that the group comprising diatom NTT2 and TPNTT3 and the other group comprising PANTT3 6 and TPNTT4 8 originated from two different events of horizontal gene transfer followed by gene duplications.

Although showing significant similarities to plastidial ATP/ ADP transporters, diatom NTT1 isoforms act as proton sym porters and mediate net uptake of diverse adenine nucleotides across the innermost plastid envelope (Ast et al., 2009). Accord ingly, the substrate spectrum and the transport mode of the ancestral red algal carrier were modified to fulfill a new function in diatom plastids. Diatom NTT2 isoforms catalyze the antiport of diverse (deoxy) nucleotides (Ast et al., 2009). Interestingly, the functional properties of diatom NTT1 and NTT2 resemble that of NTTs in intracellular bacteria. These bacteria parasitize nucleotides from the host (via interaction of functionally differ ent NTTs) and compensate their missing capacity for nucleotide synthesis (Haferkamp et al., 2004, 2006; Audia \& Winkler, 2006; Knab et al., 2011). Similarly, interaction of diatom NTT1 and NTT2 in the innermost plastid envelope was suggested to allow net gain of all substrates for DNA and RNA synthesis in the stroma (Fig. 5; Ast et al., 2009). Adenine nucleotides imported via NTT1 can in turn (in the form of ATP) drive the uptake of diverse (deoxy) nucleotides via NTT2.

This finding was in some ways unexpected because it contrasts with the strong expectation that all NTTs from plants and algae exclusively act as ATP/ADP exchangers, with a specific relevance

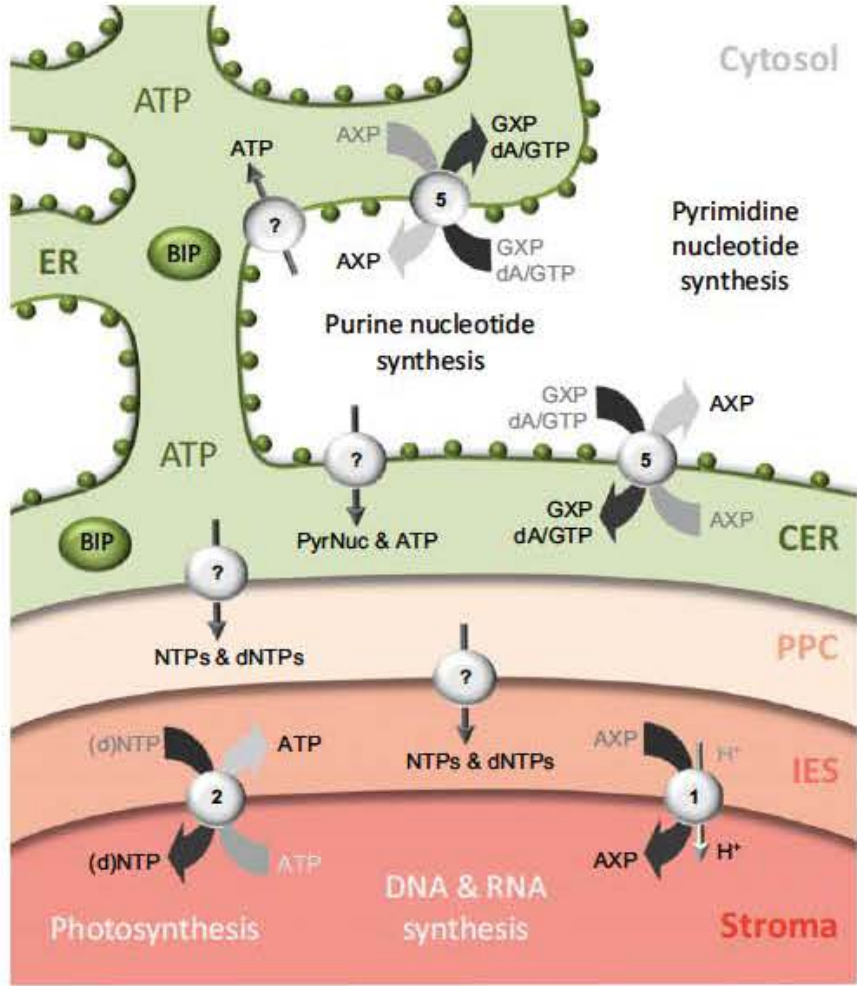

Fig. 5 Schematic illustration of the proposed interaction of the biochemically characterized nucleotide transporters (NTTs) from Phaeodactylum tricornutum. Interaction of PtNTT1 (gray circle, 1) and PtNTT2 (gray circle, 2) in the innermost plastid membrane provides substrates for DNA and RNA synthesis in the stroma. Pyrimidine and purine nucleotide synthesis takes place in the cytosol. The existence of ATP dependent reactions, such as activity of the luminal binding protein precursor (BIP) or protein folding, indicates that ATP is present in the ER and chloroplast ER (CER) lumen. However, net uptake of adenine nucleotides into the ER has generally not yet been clarified (gray circle, ?). PtNTT5 (gray circle, 5) can use luminal adenine nucleotides (AXP) to drive the uptake of guanidine nucleotides (GXP), dATP and dGTP from the cytosol. Moreover, the antiport of substrates with different phosphorylation states has an impact on the organellar energy state. Passage of nucleotides (NTPs) and deoxynucleotides (dNTPs) across the remaining plastid membranes and their entry into the periplastid compartment (PPC) and the inter envelope space (IES) as well as the entry of pyrimidine nucleotides (PyrNuc) into the ER are unclear (gray circles, ?).

for energy supply to the plastid. However, it is completely in line with the requirement for net nucleotide uptake into the complex diatom plastid. In diatoms, purine and pyrimidine nucleotides are produced exclusively in the cytosol and not, like in higher plants, mainly in the plastid stroma, which necessitates a specifi cally adapted nucleotide transport system in diatom plastids (Ast et al., 2009).

The intriguing question that arose from the previous study (Ast et al., 2009) is how nucleotides pass through the remaining plastidial membranes. The recruitment of bacterial NTTs with appropriate properties might be a valuable process to establish a transport system in the different plastid membranes that connects nucleotide synthesis in the cytosol with nucleotide consumption in the plastid stroma. A further hypothesis is that all plastid mem branes except the innermost one are selectively permeable for 
various molecules and allow more or less uncontrolled passage of nucleotides. This assumption would imply that not all diatom NTTs are involved in plastidial nucleotide uptake but fulfill diverse physiological functions.

In this study, we performed a detailed analysis of PtNTT5. In view of the low similarity of PtNTT5 to previously described NTTs (Fig. S2), it was unclear whether this protein may act as a nucleotide transporter at all. Import experiments, however, clearly demonstrated its capacity for nucleotide transport. It is specific for tri, di and monophosphorylated purine nucleotides, and dATP and dGTP are among the most favored substrates (Figs 2, S3; Tables 1, 2). Moreover, we discovered that transport activity of PtNTT5 is not dependent upon a proton gradient but on the presence of suitable exchange substrates (Fig. 3). Accord ingly, PtNTT5 represents a purine nucleotide antiporter.

The determined biochemical features of PtNTT5 are not iden tical, but closely related to those of certain bacterial and microsporidial NTTs as well as to the NTT1 isoforms of diatoms (Haferkamp et al., 2006; Audia \& Winkler, 2006; Knab et al., 2011; Ast et al., 2009; Heinz et al., 2014). This implies that not, or not only, slight modification of the transport properties, but rather other factors put specific evolutionary pressure on $P t$ NTT5 which is reflected in the distant relationship to bacterial, plastidial and microsporidial NTTs. In this context, it is impor tant to consider that PtNTT5 is apparently located in the ER membrane (Fig. 4), and therefore in an environment not typical for NTTs. It is likely that considerable structural adaptations and hence alterations in the amino acid sequence were necessary to allow appropriate targeting and embedding of an NTT in the ER membrane. Similarly, the microsporidial NTTs also exhibit bio chemical properties that are identical to or at least highly resem ble those of NTTs from bacteria or primary plastids while residing in an NTT atypical membrane. The amino acid sequences of microsporidial NTTs and PtNTT5 apparently har bor interior targeting information. Although specific motifs or domains cannot be assigned at first glance, the corresponding sequence modifications might hamper the determination of the direct ancestor of these NTTs (Fig. 1).

Phylogenetic analyses suggest that plastidial NTTs arose from an ancient chlamydial ATP/ADP transporter by horizontal gene transfer (Fig. 1; Greub \& Raoult, 2003; Schmitz Esser et al., 2004; Tyra et al., 2007). The subsequent adaptation to a new tar geting mode of the bacterial type NTT towards the plastid envelope membrane, including membrane insertion, apparently did not require substantial modifications within the amino acid sequence (Fig. S2). The most striking difference between plas tidial and chlamydial ATP/ADP transporters is the presence (ad dition) of an $\mathrm{N}$ terminal sequence extension that directs the protein to the plastid and that is cleaved off after translocation (Neuhaus et al., 1997).

To investigate the subcellular localization of PtNTT5 and, in particular, to identify possible internal targeting information, we expressed full length as well as truncated versions of this trans porter with GFP fused either $\mathrm{N}$ or $\mathrm{C}$ terminally.

Shorter versions of the $\mathrm{N}$ terminal GFP fusions ended up in the cytosol, whereas longer versions were targeted to the ER membrane (Figs 4a, S5, S10, S11). Therefore, a minimal protein length or even specific information in the $\mathrm{C}$ terminal part of $P t$ NTT5 is required for its targeting to the ER. We therefore con clude that ER targeting of PtNTT5 takes place post translationally. We suggest that a translocation pathway similar to that of $\mathrm{C}$ terminally anchored proteins might be used for post translational insertion into the ER membrane. $\mathrm{C}$ terminal or tail anchored (TA) proteins are transmembrane polypeptides that are held in the phospholipid bilayer by hydrophobic amino acids at the C terminus (Borgese et al., 2003). Direct insertion of newly synthesized TA proteins may occur in mitochondrial membranes, but also in the membranes of ER, peroxisomes or the chloroplast (Borgese et al., 2007; Borgese \& Fasana, 2011). In vitro analyses revealed the existence of different insertion path ways for TA proteins: the unassisted spontaneous integration into lipid bilayers or the energy requiring chaperone mediated path way (Borgese \& Fasana, 2011). Import competition assays sug gest that the insertion pathways of TA and multi span proteins may overlap (Otera et al., 2007; Dukanovic \& Rapaport, 2011).

In the case of $\mathrm{C}$ terminal attachment of GFP to full length or truncated versions of PtNTT5, the GFP signals always appeared in diffuse patches close to the plastid/nucleus (Figs 4c, S8, S9). Therefore, this localization is independent of the used part or length of the PtNTT5 sequence. Accordingly, no specific regions in the fusion protein, apart from the $\mathrm{C}$ terminal GFP, could be identified that might be associated with the corresponding local ization. The fluorescence pattern of the $\mathrm{C}$ terminal GFP fusions is indicative of accumulation rather than insertion in a specific membrane. It is conceivable that the diffuse GFP signals are caused by degradation of the hydrophobic P NTT5 moiety and delivery of GFP. However, western blot analyses indicate that this is not the case (Fig. S12). Alternatively, one might envision that mal targeted fusion proteins accumulate in membranous struc tures such as the Golgi apparatus or form aggregates by hydrophobic interaction in an as yet unknown compartment.

Although we cannot rule out completely the possibility that the diffuse patches reflect the correct localization, the GFP analy ses in sum suggest that PtNTT5 represents a membrane protein that is post translationally inserted into the ER.

Activity of the (deoxy) purine nucleotide shuttle PtNTT5 in the ER membrane (which is continuous with the outermost plas tid membrane) would presuppose the presence of suitable exchange substrates in the lumen. Adenine nucleotides are defi nitely present in the lumen because multiple ATP dependent reactions (e.g. protein import and folding, and ATP hydrolysis via BIP) take place in the ER (Hirschberg et al., 1998; Apt et al., 2002; Csala et al., 2007). It is not clear which proteins catalyze net adenine nucleotide loading of the ER in all eukaryotes, but members of the mitochondrial carrier family are promising can didates (Hirschberg et al., 1998; Csala et al., 2007; Leroch et al., 2008). Independent of the nature of the carriers involved, the adenine nucleotides imported into the ER can drive the antiporter PtNTT5 (Fig. 5). When substrates of different phos phorylation states are exchanged (e.g. ATP for ADP), PtNTT5 alters the organellar energy state. Interestingly, in contrast to transporters commonly known to be involved in energy 
metabolism, the substrate spectrum of PtNTT5 comprises deoxy nucleotides as additional important substrates. This fea ture can be considered an important indicator of an additional and possibly major role of PtNTT5 as a component of the nucleotide uptake system of the complex plastid: import of GTP, $\mathrm{dATP}$ and dGTP in exchange for the luminal adenine nucleotides not only alters the nucleotide composition of the ER, but also allows the passage of building blocks for DNA and RNA synthesis across the outermost plastid envelope (Fig. 5).

These findings indicate that further components of the nucleotide uptake system of diatom plastids await identification. It is unclear which factors mediate (deoxy) pyrimidine nucleotide entry into the ER/translocation across the outermost plastid envelope and how various (deoxy) nucleotides pass through membranes two and three (Fig. 5). NTT proteins can be considered as important candidates for these tasks and three of the NTT isoforms found in $P$. tricornutum have not been characterized yet. The analysis of NTT isoforms from centric diatoms might additionally help to identify common principles or discrepancies in nucleotide transport of diatoms. In fact, at least slight differences in nucleotide transport can be expected because the centric T.pseudonana possesses a different number of putative NTT isoforms from the pennate P. tricornutum (Ast et al., 2009). Interestingly, the identification of nucleotide trans porters with extraordinary properties was recently shown to pave the way for sophisticated biotechnical approaches. Heterologous expression of PtNTT2 allowed the feeding of artificial deoxy nucleotides into E. coli and finally resulted in a semi synthetic organism with an extended genetic alphabet (Malyshev et al., 2014).

\section{Acknowledgements}

We thank D. Ballert for help with the transformation and culti vation of P.tricornutum. This study was supported by the University of Konstanz, the graduate school KoRS CB, and the Deutsche Forschungsgemeinschaft DFG (SFB 969 Project A4 to P.G.K.; Reinhard Koselleck Grant NE418/16 1 to H.E.N.). We thank the Bioimaging Center (BIC), University of Konstanz, for access to the imaging core facilities.

\section{Author contributions}

L.C. designed and carried out the localization studies in diatoms and participated in the biochemical characterization and in drafting and finalizing the manuscript. A.G. carried out micro scopic analyses and participated in designing the localization studies and in drafting and finalizing the manuscript. M.A. par ticipated in the biochemical characterization. S.S E. designed and carried out the phylogenetic analyses. J.A. performed local ization studies in higher plants. H.E.N. participated in design ing the biochemical studies. P.G.K. conceived of the study and participated in designing the localization studies and in finaliz ing the manuscript. I.H. conceived of the study, drafted the manuscript and participated in the biochemical characterization and in finalizing the manuscript. All authors read and approved the manuscript.

\section{References}

Apt KE, Zaslavkaia L, Lippmeier JC, Lang M, Kilian O, Wetherbee R, Grossman AR, Kroth PG. 2002. In vivo characterization of diatom multipartite plastid targeting signals. Journal of Cell Science 115: 40614069.

Archibald JM. 2015. Genomic perspectives on the birth and spread of plastids. Proceedings of the National Academy of Sciences, USA 112: 1014710153.

Armbrust EV. 2009. The life of diatoms in the world's oceans. Nature 459: 185 192.

Ast M, Gruber A, Schmitz-Esser S, Neuhaus HE, Kroth PG, Horn M, Haferkamp I. 2009. Diatom plastids depend on nucleotide import from the cytosol. Proceedings of the National Academy of Sciences, USA 106: 36213626.

Audia JP, Winkler HH. 2006. Study of the five Rickettsia prowazekii proteins annotated as ATP/ADP translocases (Tlc): only Tlc1 transports ATP/ADP, while Tlc4 and Tlc5 transport other ribonucleotides. Journal of Bacteriology 188: 62616268.

Bailleul B, Berne N, Murik O, Petroutsos D, Prihoda J, Tanaka A, Villanova V, Bligny R, Flori S, Falconet D et al. 2015. Energetic coupling between plastids and mitochondria drives $\mathrm{CO}_{2}$ assimilation in diatoms. Nature 524: 366369.

Borgese N, Brambillasca S, Colombo S. 2007. How tails guide tail-anchored proteins to their destinations. Current Opinion in Cell Biology 19: 368375.

Borgese N, Colombo S, Pedrazzini E. 2003. The tale of tail-anchored proteins: coming from the cytosol and looking for a membrane. Journal of Cell Biology 161: 10131019

Borgese N, Fasana E. 2011. Targeting pathways of C-tail-anchored proteins. Biochimica et Biophysica Acta 1808: 937946.

Bowler C, Allen AE, Badger JH, Grimwood J, Jabbari K, Kuo A, Maheswari U, Martens C, Maumus F, Otillar RP et al. 2008. The Phaeodactylum genome reveals the evolutionary history of diatom genomes. Nature 456: 239244.

Csala M, Marcolongo P, Lizak B, Senesi S, Margittai E, Fulceri R, Magyar JE, Benedetti A, Banhegyi G. 2007. Transport and transporters in the endoplasmic reticulum. Biochimica et Biophysica Acta 1768: 13251341.

Dukanovic J, Rapaport D. 2011. Multiple pathways in the integration of proteins into the mitochondrial outer membrane. Biochimica et Biophysica Acta 1808: 971980

Emanuelsson O, Brunak S, von Heine G, Nielsen H. 2007. Locating proteins in the cell using TargetP, SignalP and related tools. Nature Protocols 2: 953971.

Falkowski PG, Oliver MJ. 2007. Mix and match: how climate selects phytoplankton. Nature Reviews Microbiology 5: 813819.

Geigenberger P, Stamme C, Tjaden J, Schulze A, Quick WP, Betsche T, Kersting HJ, Neuhaus HE. 2001. Tuber physiology and properties of starch from tubers of transgenic potato plants with altered plastidic adenylate transporter activity. Plant Physiology 125: 16671678.

Gould SB, Waller RF, McFadden GI. 2008. Plastid evolution. Annual Review of Plant Biology 59: 491517.

Grefen C, Donald N, Hashimoto K, Kudla J, Schumacher K, Blatt MR. 2010. A ubiquitin-10 promoter-based vector set for fluorescent protein tagging facilitates temporal stability and native protein distribution in transient and stable expression studies. Plant Journal 64: 355365.

Greub G, Raoult D. 2003. History of the ADP/ATP-translocase-encoding gene, a parasitism gene transferred from a Chlamydiales ancestor to plants 1 billion years ago. Applied and Environment Microbiology 69: 55305535.

Gruber A, Kroth PG. 2014. Deducing intracellular distributions of metabolic pathways from genomic data. Methods in Molecular Biology 1083: 187211.

Gruber A, Rocap G, Kroth PG, Armbrust EV, Mock T. 2015. Plastid proteome prediction for diatoms and other algae with secondary plastids of the red lineage. Plant Journal 81: 519528.

Gruber A, Vugrinec S, Hempel F, Gould SB, Maier UG, Kroth PG. 2007. Protein targeting into complex diatom plastids: functional characterisation of a specific targeting motif. Plant Molecular Biology 64: 519530. 
Haferkamp I, Schmitz-Esser S, Linka N, Urbany C, Collingro A, Wagner M, Horn M, Neuhaus HE. 2004. A candidate $\mathrm{NAD}^{+}$transporter in an intracellular bacterial symbiont related to Chlamydiae. Nature 432: 622625 .

Haferkamp I, Schmitz-Esser S, Wagner M, Neigel N, Horn M, Neuhaus HE. 2006. Tapping the nucleotide pool of the host: novel nucleotide carrier proteins of Protochlamydia amoebophila. Molecular Microbiology 60: 1534 1545.

Heinz E, Hacker C, Dean P, Mifsud J, Goldberg AV, Williams TA, Nakjang S, Gregory A, Hirt RP, Lucocq JM etal. 2014. Plasma membrane-located purine nucleotide transport proteins are key components for host exploitation by microsporidian intracellular parasites. PLoS Pathogens 10: e1004547.

Hirschberg CB, Robbins PW, Abeijon C. 1998. Transporters of nucleotide sugars, ATP, and nucleotide sulfate in the endoplasmic reticulum and Golgi apparatus. Annual Review of Biochemistry 67: 4969.

Katoh K, Standley DM. 2013. MAFFT multiple sequence alignment software version 7: improvements in performance and usability. Molecular Biology and Evolution 30: 772780.

Keeling PJ. 2013. The number, speed, and impact of plastid endosymbioses in eukaryotic evolution. Annual Review of Plant Biology 64: 583607.

Kilian O, Kroth PG. 2005. Identification and characterization of a new conserved motif within the presequence of proteins targeted into complex diatom plastids. Plant Journal 41: 175183.

Kirchberger S, Tjaden J, Neuhaus HE. 2008. Characterization of the Arabidopsis Brittle1 transport protein and impact of reduced activity on plant metabolism. Plant Journal 56: 5163.

Knab S, Mushak TM, Schmitz-Esser S, Horn M, Haferkamp I. 2011. Nucleotide parasitism by Simkania negevensis (Chlamydiae). Journal of Bacteriology 193: 225235.

Krause DC, Winkler HH, Wood DO. 1985. Cloning and expression of the Rickettsia prowazekii ADP/ATP translocator in Escherichia coli. Proceedings of the National Academy of Sciences, USA 82: 30153019.

Kroth PG. 2002. Protein transport into secondary plastids and the evolution of primary and secondary plastids. International Review of Cytology 221: 191255.

Kroth PG. 2007. Genetic transformation: a tool to study protein targeting in diatoms. Methods in Molecular Biology 390: 257267.

Laemmli UK. 1970. Cleavage of structural proteins during the assembly of the head of bacteriophage T4. Nature 227: 680685 .

Leroch M, Kirchberger S, Haferkamp I, Wahl M, Neuhaus HE, Tjaden J. 2005. Identification and characterization of a novel plastidic adenine nucleotide uniporter from Solanum tuberosum. Journal of Biological Chemistry 280: 17992 18000 .

Leroch M, Neuhaus HE, Kirchberger S, Zimmermann S, Melzer M, Gerhold J, Tjaden J. 2008. Identification of a novel adenine nucleotide transporter in the endoplasmic reticulum of Arabidopsis. Plant Cell 20: 438451.

Linka N, Hurka H, Lang BF, Burger G, Winkler HH, Stamme C, Urbany C, Seil I, Kusch J, Neuhaus HE. 2003. Phylogenetic relationship of nonmitochondrial nucleotide transport proteins in bacteria and eukaryotes. Gene 306: 2735 .

Malyshev DA, Dhami K, Lavergne T, Chen T, Dai N, Foster JM, Correa IR Jr, Romesberg FE. 2014. A semi-synthetic organism with an expanded genetic alphabet. Nature 509: 385388.

Mohlmann T, Tjaden J, Schwoppe C, Winkler HH, Kampfenkel K, Neuhaus HE. 1998. Occurrence of two plastidic ATP/ADP transporters in Arabidopsis thaliana $\mathrm{L}$. Molecular characterisation and comparative structural analysis of similar ATP/ADP translocators from plastids and Rickettsia prowazekii. European Journal of Biochemistry 252: 353359.

Moran NA. 2002. Microbial minimalism: genome reduction in bacterial pathogens. Cell 108: 583.

Moulder JW. 1991. Interaction of chlamydiae and host cells in vitro. Microbiological Reviews 55: 143190.

Nelson BK, Cai X, Nebenfuhr A. 2007. A multi-color set of in vivo organelle markers for colocalization studies in Arabidopsis and other plants. Plant Journal 51: 11261136

Neuhaus HE, Thom E, Mohlmann T, Steup M, Kampfenkel K. 1997. Characterization of a novel eukaryotic ATP/ADP translocator located in the plastid envelope of Arabidopsis thaliana L. Plant Journal 11: 7382.
Nicholas KB, Nicholas HB Jr, Deerfield DW II. 1997. GeneDoc: analysis and visualization of genetic variation. Embnet.news 4: 14 .

Otera H, Taira Y, Horie C, Suzuki Y, Suzuki H, Setoguchi K, Kato H, Oka T, Mihara K. 2007. A novel insertion pathway of mitochondrial outer membrane proteins with multiple transmembrane segments. Journal of Cell Biology 179: 13551363.

Reinhold T, Alawady A, Grimm B, Beran KC, Jahns P, Conrath U, Bauer J, Reiser J, Melzer M, Jeblick W et al. 2007. Limitation of nocturnal import of ATP into Arabidopsis chloroplasts leads to photooxidative damage. Plant Journal 50: 293304.

Reiser J, Linka N, Lemke L, Jeblick W, Neuhaus HE. 2004. Molecular physiological analysis of the two plastidic ATP/ADP transporters from Arabidopsis thaliana. Plant Physiology 136: 35243536.

Schmitz-Esser S, Haferkamp I, Knab S, Penz T, Ast M, Kohl C, Wagner M, Horn M. 2008. Lawsonia intracellularis encodes a functional rickettsia-like ATP/ADP translocase for host exploitation. Journal of Bacteriology 190: 5746 5752.

Schmitz-Esser S, Linka N, Collingro A, Beier CL, Neuhaus HE, Wagner M, Horn M. 2004. ATP/ADP translocases: a common feature of obligate intracellular amoebal symbionts related to chlamydia and rickettsia. Journal of Bacteriology 186: 683691.

Tamura K, Stecher G, Peterson D, Filipski A, Kumar S. 2013. MEGA6: molecular evolutionary genetics analysis version 6.0. Molecular Biology and Evolution 30: 2725 2729.

Thompson JD, Gibson DJ, Plewniak F, Jeanmougin F, Higgins DG. 1997. The CLUSTAL X windows interface: flexible strategies for multiple sequence alignment aided by quality analysis tools. Nucleic Acids Research 25: 4876 4882.

Tjaden J, Mohlmann T, Kampfenkel K, Henrichs G, Neuhaus HE. 1998a. Altered plastidic ATP/ADP-transporter activity influences potato (Solanum tuberosum) tuber morphology, yield and composition of tuber starch. Plant Journal 16: 531540.

Tjaden J, Schwoppe C, Mohlmann T, Quick WP, Neuhaus HE. 1998b. Expression of the plastidic ATP/ADP transporter gene in Escherichia coli leads to a functional adenine nucleotide transport system in the bacterial cytoplasmic membrane. Journal of Biological Chemistry 273: 96309636.

Tjaden J, Winkler HH, Schwoppe C, van der Laan M, Mohlmann T, Neuhaus HE. 1999. Two nucleotide transport proteins in Chlamydia trachomatis, one for net nucleoside triphosphate uptake and the other for the transport of energy. Journal of Bacteriology 181: 11961202.

Tsaousis AD, Kunji ER, Goldberg AV, Lucocq JM, Hirt RP, Embley TM. 2008. A novel route for ATP acquisition by the remnant mitochondria of Encephalitozoon cuniculi. Nature 453: 553556.

Tyra HM, Linka M, Weber AP, Bhattacharya D. 2007. Host origin of plastid solute transporters in the first photosynthetic eukaryotes. Genome Biology 8: R212.

Witz S, Jung B, Furst S, Mohlmann T. 2012. De novo pyrimidine nucleotide synthesis mainly occurs outside of plastids, but a previously undiscovered nucleobase importer provides substrates for the essential salvage pathway in Arabidopsis. Plant Cell 24: 15491559.

Zaslavskaia LA, Lippmeier JC, Kroth PG, Grossman A, Apt KE. 2000. Transformation of the diatom Phaeodactylum tricornutum (Bacillariophyceae) with a variety of selectable marker and reporter genes. Journal of Phycology 36: 379386.

Zrenner R, Stitt M, Sonnewald U, Boldt R. 2006. Pyrimidine and purine biosynthesis and degradation in plants. Annual Review of Plant Biology 57: 805836 .

\section{Supporting Information}

Additional Supporting Information may be found online in the Supporting Information tab for this article:

Fig. S1 Exon-intron structure of diatom NTTs. 
Fig. S2 Amino acid sequence alignment of biochemically charac terized NTTs.

Fig. S3 Time dependent uptake of purine nucleotides via PtNTT5.

Fig. S4 Cellular localization of PtBIP::GFP in Phaeodactylum tricornutum cells.

Fig. S5 Cellular localization of PtNTT5 with N terminal GFP in plant cells.

Fig. S6 Cellular localization of PtNTT5 with C terminal GFP in plant cells.

Fig. S7 Schematic illustration of GFP fusion proteins of full length and truncated PtNTT5.

Fig. S8 Localization of PtNTT5 in Phaeodactylum tricornutum (C terminal deletion and $\mathrm{C}$ terminal GFP fusion).

Fig. S9 Localization of PtNTT5 in Phaeodactylum tricornutum ( $\mathrm{N}$ terminal deletion and $\mathrm{C}$ terminal GFP fusion).
Fig. S10 Localization of PtNTT5 in Phaeodactylum tricornutum (C terminal deletion and $\mathrm{N}$ terminal GFP fusion).

Fig. S11 Localization of PtNTT5 in Phaeodactylum tricornutum (C terminal deletion and $\mathrm{N}$ terminal GFP fusion).

Fig. S12 Expression analysis of selected $\mathrm{C}$ terminal and $\mathrm{N}$ terminal GFP fusions of PtNTT5.

Table S1 Oligonucleotides used to generate PtNTT5 GFP fusion constructs

Table S2 GC content of diatom NTTs

Table S3 Amino acid similarities of diverse functionally charac terized NTTs (in \%)

Please note: Wiley Blackwell are not responsible for the content or functionality of any Supporting Information supplied by the authors. Any queries (other than missing material) should be directed to the New Phytologist Central Office. 